\title{
Patterning of organic thin film transistors by oxygen plasma etch
}

\author{
Soeren Steudel, ${ }^{\text {a),b) }}$ Kris Myny, Stijn De Vusser, ${ }^{\text {b) }}$ Jan Genoe, and Paul Heremans ${ }^{\text {b) }}$ \\ Polymer and Molecular Electronics, IMEC, Kapeldreef 75, 3001 Leuven, Belgium
}

(Received 24 July 2006; accepted 15 September 2006; published online 31 October 2006)

\begin{abstract}
All applications of organic thin film transistors require patterning of the organic thin film to achieve a low off current and to prevent cross talk between neighboring transistors. A common method for patterning consists of using a protective layer and etching the uncovered small molecule film by oxygen plasma. One of the handicaps of this process is the observed degradation of the transistor characteristics. By varying the resist overlap, the authors show that the main cause of this performance degradation is, in fact, a far-reaching underetch of the oxygen plasma which can be overcome by choosing the right geometry of the resist pattern. (C) 2006 American Institute of Physics. [DOI: 10.1063/1.2374679]
\end{abstract}

In the last decade, the performance of organic thin film transistor (OTFT) improved rapidly. Two potential applications motivate the work on OTFTs. On one hand, there is a great interest in using OTFTs in active matrix pixel drivers for displays, possibly on flexible substrates. Another focus of OTFT research are low-cost integrated circuits, in particular, passive radio frequency identification (rf-ID) tags.

The best performance in OTFTs has been achieved with evaporated small molecules, whereby the reported hole mobilities exceeded $1 \mathrm{~cm}^{2} / \mathrm{V} \mathrm{s}$ in case of evaporated pentacene, ${ }^{1}$ overtaking amorphous silicon. For all applications of OTFTs it is necessary to limit cross talk between neighboring transistors and to achieve a low off current. Specifically for OTFTs used as pixel driver in displays, an off current below $10 \mathrm{pA}$ is required. ${ }^{2}$ Otherwise, the hold capacitance in a pixel needs to be unreasonably large. In the case of circuits, it is not so much the absolute off current that is important, but rather the on/off ratio that should be at least $10^{3}$. Furthermore, it is advantageous to use reasonably small values of width to length ratio $(W / L)$ of the OTFTs to reduce the power and area consumptions. This results in a requirement of absolute low off current for circuits as well.

Currents in the range of a few picoamperes cannot be reached without patterning the organic semiconductor layer such that the gate extends beyond the active area. Depending on the onset voltage $V_{\text {on }}$ and the geometry of the OTFT, devices based on pentacene typically have an off current in the range of $1-10 \mathrm{nA}$.

Several methods of patterning small molecules can be found in the literature, i.e., deposition through a shadow mask, ${ }^{1}$ integrated shadow mask, ${ }^{3}$ or patterned growth. ${ }^{4}$ One of the most common methods for patterning consists of depositing either a water-soluble resist ${ }^{5}$ or a protective parylene layer followed by a regular photoresist ${ }^{6}$ on top of the organic small molecule layer, which is used as an etch mask. Subsequently the small molecule layer is etched by an oxygen plasma.

The advantage of this process compared to other ways of patterning consists in the remaining thick protective resist layer, enabling further encapsulation steps. Furthermore, this

\footnotetext{
${ }^{\text {a) }}$ Electronic mail: ssteudel@imec.be

${ }^{b)}$ Also at Department of Electrical Engineering of K.U. Leuven, Leuven 3000, Belgium.
}

process is compatible with printing processes as well as photolithographic processes.

One of the handicaps of this process is the observed degradation of the transistor performance. Recently, the first active matrix organic light emitting diode (OLED) display with OTFTs as pixel driver and using water-soluble resist to pattern pentacene transistors has been published ${ }^{7}$. The curves published in that article as well as other articles ${ }^{4,8}$ using this patterning technique show a subthreshold slope as low as $1 \mathrm{~V} / \mathrm{dec}$ for a gate capacitance equivalent of $100 \mathrm{~nm} \mathrm{SiO}{ }_{2}$, although devices made by shadow masking or manually scratching the pentacene layer around the transistor result typically in subthreshold slopes of as low as $0.2 \mathrm{~V} / \mathrm{dec}$.

We observed that transistors with large $W / L$ patterned with water-soluble resist and oxygen plamsa etch do hardly suffer a degraded subthreshold slope. This observation led us to conclude that it is not the water-soluble resist that caused the degradation of the transistor characteristics as observed when patterning transistors with a small $W / L$, rather processing issues pronounced by the geometry of the transistor design are the suspected cause.

We therefore fabricated bottom-gate transistor structures as depicted in Fig. 1 with various $W / L$ (5000/10, 1000/10, 200/10, 100/10, and 20/10) and different sizes of the resist overlap $d$ extending 15,50 , and $200 \mu \mathrm{m}$ beyond the transistor structure. To achieve a high mobility and low contact resistance, we deposited octadecyltrichlorosilane on the sputtered $\mathrm{SiO}_{2}$ dielectric and octadecylthiol on the $\mathrm{Au}$ sourcedrain contacts. Subsequently the $50 \mathrm{~nm}$ pentacene was deposited under high vacuum at $0.25 \AA / \mathrm{s}$ at a substrate temperature of $68^{\circ} \mathrm{C}$.

The $I_{\mathrm{DS}}-V_{\mathrm{GS}}$ curve of an unpatterned device can be seen in Fig. 2. In addition, the $I_{\mathrm{DS}}-V_{\mathrm{GS}}$ curve of the same device after manually scratching the pentacene layer around the device is added to demonstrate that a pentacene OTFT can be completely switched off down to the level of leakage current $(J)$ through the dielectric (i.e., leakage at $1 \mathrm{MV} / \mathrm{cm} \rightarrow J_{\text {sputtered } \mathrm{SiO}_{2}} \sim 10^{-10} \mathrm{~A} / \mathrm{mm}^{2}$ ). This is, however, only the case if the intrinsic doping is low enough.

On top of the pentacene film, a solution of a photosensitive cross-linkable polymer based on functionalized polyvinyl alcohol, similar to Kane et al., ${ }^{5}$ is spincoated. This results into a resist thickness of $\sim 1 \mu \mathrm{m}$. An $80 \mathrm{~s}$ bakeout on a hot plate at $90{ }^{\circ} \mathrm{C}$ follows. We illuminate the resist in the 


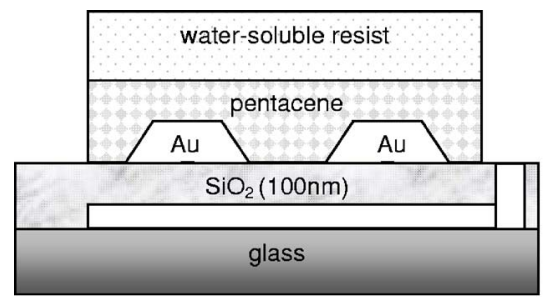

(a)

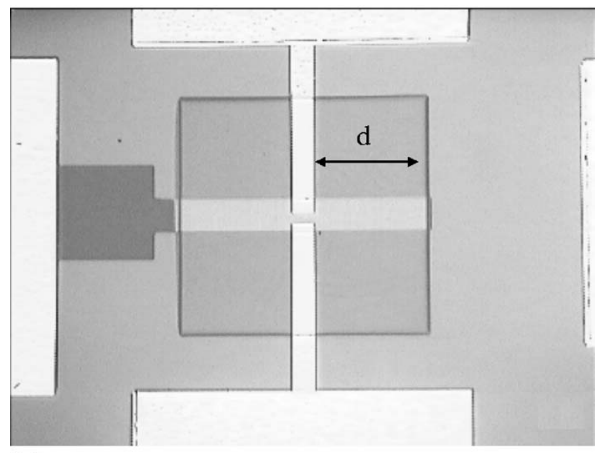

(b)

FIG. 1. OTFT: (a) schematic and (b) photograph $(W / L=20 / 10)$.

active areas with $G$-line illumination with a Karl Süss MA6. The resist is developed in $\mathrm{H}_{2} \mathrm{O}$ for $45 \mathrm{~s}$. The pentacene that is not covered by the resist is then removed in oxygen plasma $(6 \mathrm{~min}$ at $100 \mathrm{~W})$.

The $I_{\mathrm{DS}}-V_{\mathrm{GS}}$ of the devices with $5000 / 10,200 / 10$, and 20/10 can be seen in Figs. 3(a)-3(c). It is obvious from all three graphs that the devices with a small resist overlap have a degraded subthreshold slope, a higher onset voltage $V_{\text {on }}$, and a higher $V_{T}$ as compared to OTFTs with a large overlap. Transistors with the maximum overlap of $200 \mu \mathrm{m}$ all have the same $V_{\text {on }}$, independent of their $W / L$. In addition, the mobility of the transistors is significantly degraded as a function of the overlap in small transistors as compared to large transistors where the mobility stays nearly constant. Both phenomena can be explained if we assume that the oxygen plasma causes far-reaching lateral damage to the pentacene and the dielectric, even below the protective resist layer, resulting in the formation of negatively charged states and therefore shifting the threshold voltage to more positive values. ${ }^{9}$ The amount of oxidation of the pentacene layer gradually decreases in the pentacene regions that are further away from the resist edge. Therefore if we use a large $W / L$, which is mostly realized by interdigitated finger structures, some regions at the fringe might be degraded and therefore

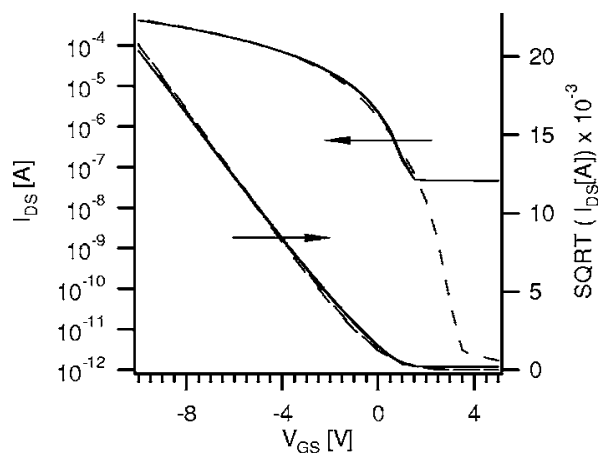

FIG. 2. $I_{\mathrm{DS}}-V_{\mathrm{GS}}$ curve of an OTFT (solid line-without patterning; dotted line-with patterning). etch of the organic film outside the active area. Investigations
line-with patterning).
Downloaded 07 Nov 2006 to 146.103.254.11. Redistribution subject to AlP license or copyright, see http://apl.aip.org/apl/copyright.jsp
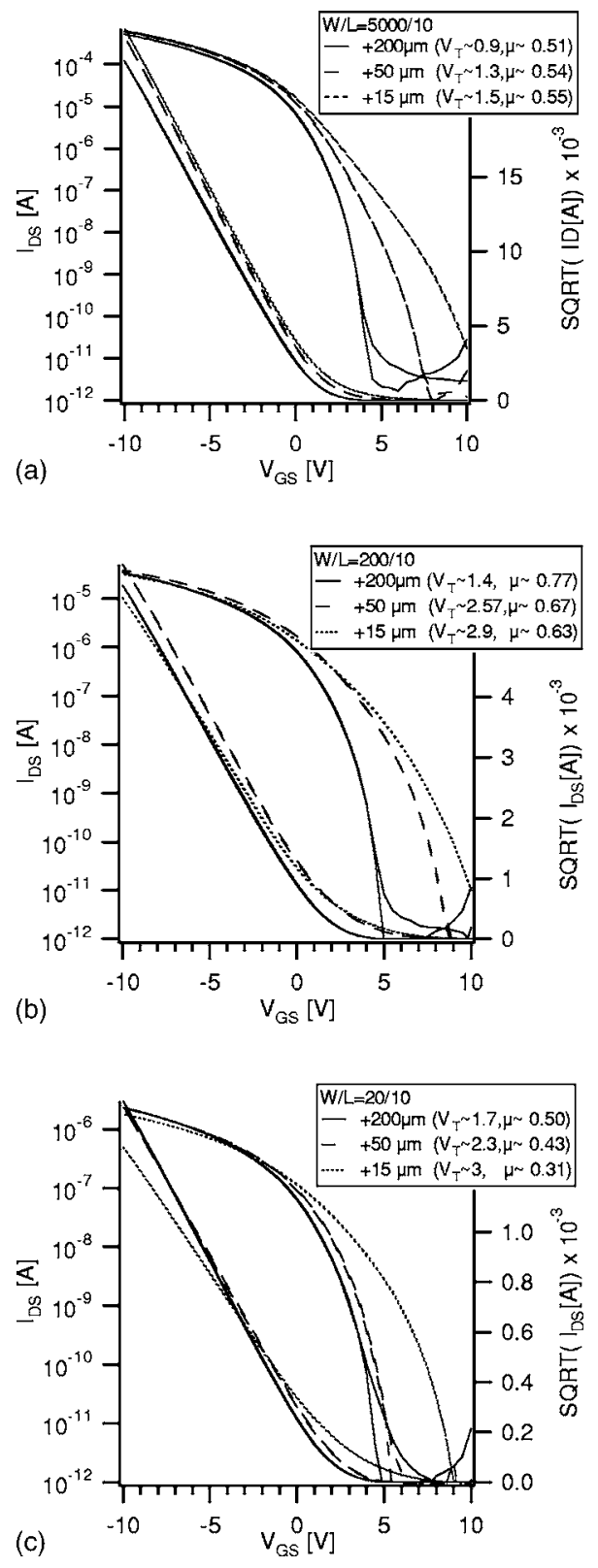

FIG. 3. $I_{\mathrm{DS}}-V_{\mathrm{GS}}$ curve of OTFTs after patterning with different resist overlaps: (a) $(W / L=5000 / 10$, (b) $W / L=200 / 10$, and (c) $W / L=20 / 10$.

contribute to a higher onset voltage, but the majority of the transistor channel still has the same mobility and threshold voltage. When using small transistors, however, the whole transistor channel is affected, causing a more severe shift in threshold voltage.

This finding has consequences for the design of OTFTs using this patterning technique, making a resist overlap of at least $100 \mu \mathrm{m}$ necessary. Considering circuits, without extension of the patterning area, we get a larger variation in threshold voltage between different transistors and therefore reduce the stability of our circuits. ${ }^{10}$ Furthermore, patterning by oxygen plasma etch should not be considered for display applications, where the area of the transistors for addressing the OLED pixel is severely limited to achieve a good aperture ratio. Circuits in sensor readouts or rf-ID tags are less constrained regarding area consumption.

In summary, we have optimized a patterning technique for small molecule layers that is based on oxygen plasma 
into the reliability of this process have shown that farreaching oxidation below the etch mask severely degrades the transistor performance. Degradation can be prevented by using the appropriate design rules shown in this letter.

This work was partially supported by the Europeanfunded Integrated Project POLYAPPLY (IST No. 507143).

${ }^{1}$ T. W. Kelley, D. V. Muyres, P. F. Baude, T. P. Smith, and T. D. Jones, Mater. Res. Soc. Symp. Proc. 771, L.6.5.1 (2003).

${ }^{2}$ H. E. A. Huitema, G. H. Gelinck, J. B. P. H. van der Putten, K. E. Kuijk, K. M. Hart, E. Cantatore, and D. M. de Leeuw, Adv. Mater. (Weinheim, Ger.) 14, 1201 (2002).

${ }^{3}$ S. De Vusser, S. Steudel, K. Myny, J. Genoe, and P. Heremans, Appl. Phys. Lett. 88, 103501 (2006).
${ }^{4}$ S. Steudel, D. Janssen, S. Verlaak, J. Genoe, and P. Heremans, Appl. Phys. Lett. 85, 5550 (2004).

${ }^{5}$ M. G. Kane, J. Campi, M. S. Hammond, F. P. Cuomo, B. Greenings, C. D. Sheraw, J. A. Nichols, D. J. Gundlach, J. R. Huang, C. C. Kuo, L. Jia, H. Klauk, and T. N. Jackson, IEEE Electron Device Lett. 21, 534 (2000).

${ }^{6}$ I. Kymissis, C. D. Dimitrakopoulos, and S. Purushothaman, J. Vac. Sci. Technol. B 20, 956 (2002).

${ }^{7}$ L. Zhou, S. Park, B. Bai, J. Sun, S.-C. Wu, T. N. Jackson, S. Nelson, D. Freeman, and Y. Hong, IEEE Electron Device Lett. 26, 640 (2005).

${ }^{8}$ C. D. Sheraw, J. A. Nichols, D. J. Gundlach, J. R. Huang, C. C. Kuo, H. Klauk, T. N. Jackson, M. G. Kane, J. Campi, F. P. Cuomo, and B. K. Greening, Tech. Dig. - Int. Electron Devices Meet. 2000, 619.

${ }^{9}$ A. Wang, I. Kymissis, V. Bulovic, and A. I. Akinwande, Tech. Dig. - Int. Electron Devices Meet. 2004, 381.

${ }^{10} \mathrm{~S}$. De Vusser, J. Genoe, and P. Heremans, IEEE Trans. Electron Devices 53, 601 (2006). 